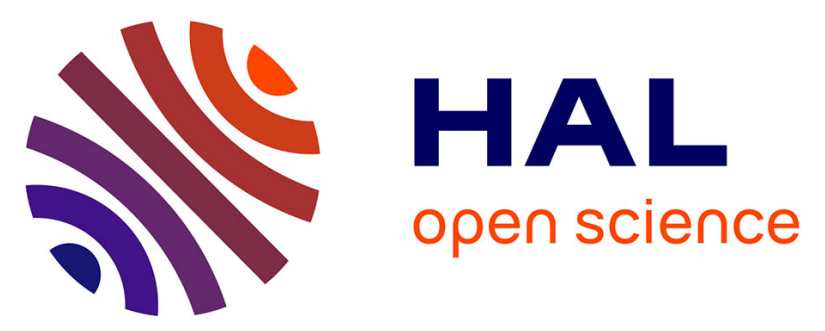

\title{
Les fouilles de la grotte-abri de Peyrazet (Creysse, Lot): nouvelles données pour le Tardiglaciaire quercinois
}

\author{
Mathieu Langlais, Véronique Laroulandie, Laurent Bruxelles, Pierre Chalard, \\ David Cochard, Sandrine Costamagno, Géraldine Delfour, Delphine Kuntz, \\ Olivier Le Gall, Jean-Marc Pétillon, et al.
}

\section{To cite this version:}

Mathieu Langlais, Véronique Laroulandie, Laurent Bruxelles, Pierre Chalard, David Cochard, et al.. Les fouilles de la grotte-abri de Peyrazet (Creysse, Lot): nouvelles données pour le Tardiglaciaire quercinois. Bulletin de la Société préhistorique française, 2009, 106 (1), pp.150-152. hal-02017906

\section{HAL Id: hal-02017906 \\ https://hal-univ-tlse2.archives-ouvertes.fr/hal-02017906}

Submitted on 22 Nov 2019

HAL is a multi-disciplinary open access archive for the deposit and dissemination of scientific research documents, whether they are published or not. The documents may come from teaching and research institutions in France or abroad, or from public or private research centers.
L'archive ouverte pluridisciplinaire HAL, est destinée au dépôt et à la diffusion de documents scientifiques de niveau recherche, publiés ou non, émanant des établissements d'enseignement et de recherche français ou étrangers, des laboratoires publics ou privés. 


\section{Les fouilles de la grotte-abri de Peyrazet (Creysse, Lot) : nouvelles données pour le Tardiglaciaire quercinois}

À l'interface du Massif central et de la plaine aquitaine, le Haut-Quercy, parcouru par la Dordogne lotoise, forme un domaine bien connu historiographiquement pour ses découvertes archéologiques attribuées à la fin du Paléolithique (Lorblanchet, 1972). Toutefois, mis à part le sondage réalisé à l'abri Murat (séquence MagdalénienAzilien; Lorblanchet, 1989 et 1996) et la fouille de Pégourié pour l'Azilien (Séronie-Vivien dir., 1995) effectués tous deux dans les années 1980, le Tardiglaciaire de cette région demeure essentiellement documenté par d'abondantes collections lithiques et osseuses provenant de fouilles anciennes du début du $X^{\circ}{ }^{\circ}$ siècle (Bergougnoux, 1887; Viré, 1909 et 1912; Lemozi, 1924; Viré et Teulière, 1927). L'opération de fouilles programmées conduite à la grotte-abri de Peyrazet a pour objectif de documenter, à l'aide de méthodes récentes, les modes de vie des derniers chasseurs de Renne dans cette région. Ce travail prend place au sein d'une dynamique de recherches menées sur les groupes humains du Tardiglaciaire dans le Sud-Ouest de la France. II s'appuie sur une équipe pluridisciplinaire, dont les membres sont rattachés aux laboratoires TRACES-UMR 5608, université de Toulouse II et PACEA-UMR5199, université de Bordeaux, et sur des travaux de synthèse récemment aboutis $(e . g$. Pétillon, 2006; Langlais, 2007; Costamagno et al., 2008).

La grotte-abri de Peyrazet a été découverte par L. Cadilhac à la fin des années quatre-vingt-dix lors de prospections spéléologiques. À l'aplomb du porche, la chute d'un arbre ayant mis au jour une coupe et des vestiges archéologiques, un sondage d'évaluation fut réalisé en 1991 par P. Chalard. Ces premiers travaux ont mis en évidence une stratigraphie matérialisée par différentes couches, plus ou moins riches en vestiges lithiques et osseux, qui témoignent de plusieurs occupations de la fin du Paléolithique supérieur (Chalard, 1992). Mis à part quelques tessons de céramique récoltés en surface dans le fond de l'abri, aucun autre indice d'occupations holocènes n'a été décelé. Le réexamen du matériel de 1991 a permis de confirmer l'attribution d'une large partie du matériel au Magdalénien supérieur (Langlais et Laroulandie dir.,
2007). Lors de la campagne de terrain qui s'est déroulée en juin 2008 , le site a été aménagé et les fouilles ont débuté à partir du secteur entamé en 1991.

Au cours de cette campagne (Langlais et Laroulandie dir., 2008), un ensemble supérieur attribuable à l'Azilien (ancien?) a été mis en évidence dans un secteur où ces niveaux n'avaient pas été touchés par la chute de l'arbre de 1991. Quant à l'ensemble inférieur de 1991, rattaché au Magdalénien supérieur, il a été complété par de nouvelles découvertes. Le site a été replacé dans son cadre géomorphologique (L. B.). L'élargissement de la coupe frontale sur environ $4 \mathrm{~m}$ a permis de mener une première étude géoarchéologique du remplissage (G. D. avec l'aide d'A. Q.). Le mobilier archéologique se compose de déchets provenant de l'exploitation de ressources animales et minérales. Parmi les Ongulés, le Renne (S. C.) est l'espèce dominante pour laquelle une étude biométrique (D. K.) nécessitera un corpus élargi. Quelques restes de Cheval sont documentés dans l'ensemble inférieur et de rares éléments de Cerf et Chevreuil dans l'ensemble supérieur (S. C.). Les microvertébrés sont abondants et variés : aux rongeurs (D. C.) et oiseaux (V. L.) sont associés de nombreux restes d'ichtyofaune (O. L. G.), Chevesne et Truite principalement (Le Gall, 1999, p. 192193). Plusieurs indices squeletto-chronologiques (vertèbres de poisson, os de jeunes ongulés) vont dans le sens d'occupations centrées sur la bonne saison, comme c'est généralement le cas dans le Quercy (Le Gall et al., 2007). Concernant l'industrie lithique (M. L.), les niveaux supérieurs (c.2-c.3) ont livré des pointes à dos (fig. 1), quelques « grandes» lames rectilignes retouchées et des grattoirs sur éclat, permettant de proposer l'attribution de cet ensemble à l'Azilien (ancien?). Pour l'ensemble inférieur, l'outillage, principalement sur lames, est dominé par les burins, tandis que les lamelles sont dévolues aux microlithes (lamelles à dos simples). Les matières premières siliceuses (P. C.) proviennent, pour une part, d'un approvisionnement régional (silex du Bajocien, notamment) et pour le reste d'affleurements orientaux (Sénonien du Périgord, Campanien de Belvès ou silex du type «Grain de Mil» originaire de Saintonge, soit à environ $200 \mathrm{~km}$ du site). L'exploitation de galets (M. L.) est documentée à travers des macro-outils, en quartz et granite notamment, et un débitage d'éclats en basalte, lequel est rarement souligné pour cette période dans cette
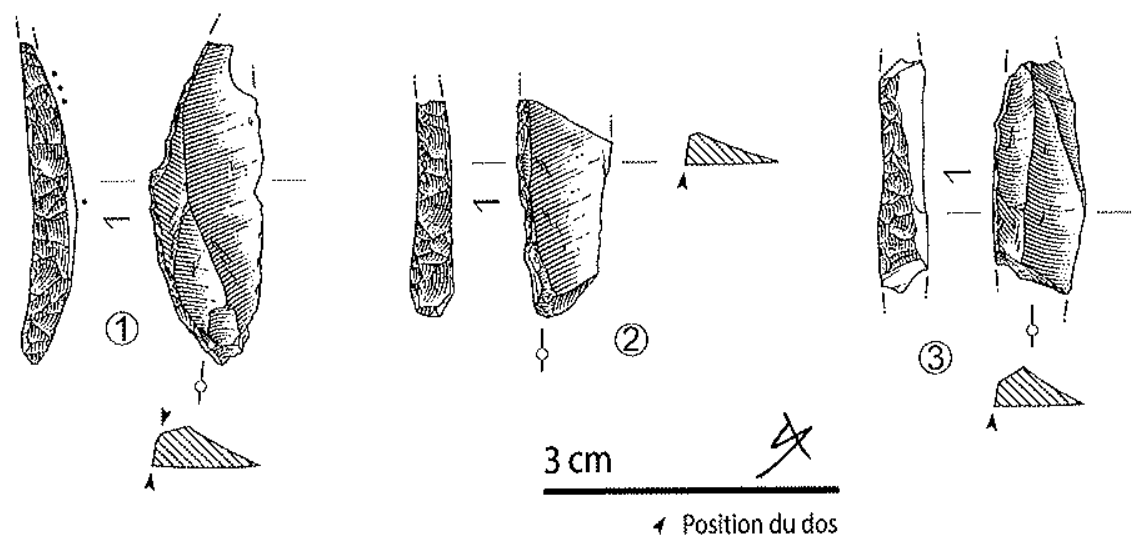

Fig. 1 - Exemples de pointes à dos de l'ensemble supérieur azilien (dessins S. Ducasse). 


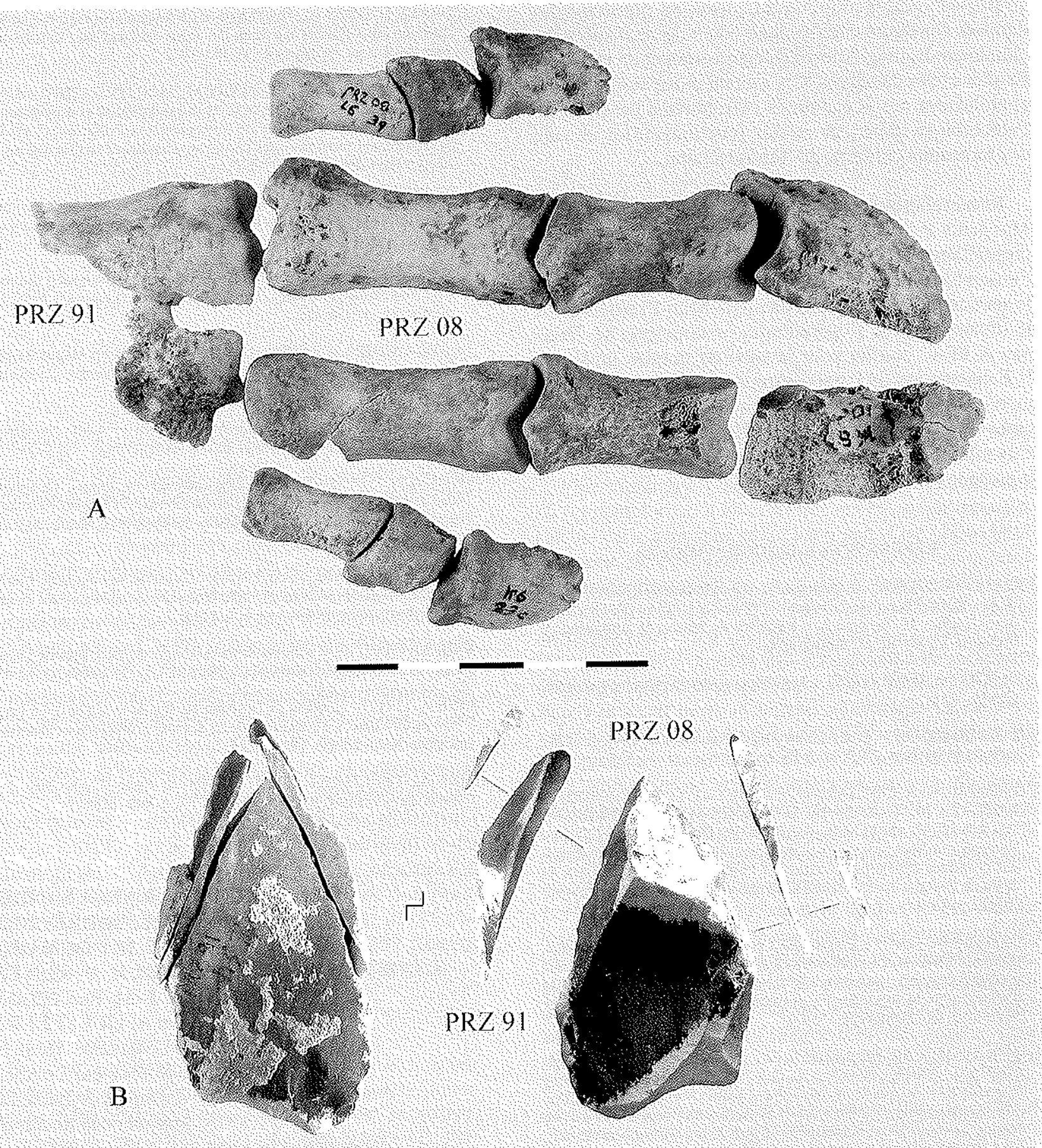

Fig. 2- Exemples de comexions anatomiques (A : pate de Renne) el de remontages litliques (B : burin et chutes) entre des vestiges découverts en 1991 et en 2008 (DAO V. L. et M. L.).

région. L'approvisionnement en ces matériaux a pu se faire sur les plages de la Dordogne qui coule à quelques centaines de mètres du site. Parmi les rares vestiges d'industrie osseuse mis au jour (J.-M. P.), nous pouvons noter la présence d'une originale double pointe « crantée» en bois de Cervidé, d'une petile bipointe (possible hameçon?) ainsi que de quelques déchets de débitage montrant la technique du rainurage longitudinal multiple, typique du Magdalénien. La réalisation de nombreux remontages fauniques (S. C. et V. L.; fracturés sur os frais et connexions anatomiques) et lithiques (M. L. et V. L., fig. 2) témoigne d'une bonne conservation de ce secteur, limité par deux grandes dalles.

À la lueur de ces premiers résultats, plusieurs axes de recherche peuvent être envisagés. À l'échelle du Quercy, la séquence de Peyrazet fait écho à celle de l'abri Murat localisé à quelques kilomètres (Lorblanchet, 1989 et 1996; Ballista, 2006) ouvrant ainsi des perspectives de comparaison microrégionale. Plus largement, nos travaux alimentent la discussion sur I'évolution des 
stratégies socio-économiques mises en place par les derniers chasseurs-cueilleurs au cours du réchauffement climatique Bølling-Allerød. L'élargissement de la fenêtre fouillée et des datations ${ }^{14} \mathrm{C}$ s'avèrent indispensables pour appréhender ces occupations saisonnières de la fin du Paléolithique supérieur et leur fonctionnement au sein des territoires culturels du Tardiglaciaire.

Remerciements : Les auteurs tiennent à remercier Messieurs P.-L. Lasfargues et P. Jardel, respectivement propriétaires de l'abri et de la parcelle d'accès au site, l'ensemble des fouilleurs ayant participé à la campagne, le service régional de l'Archéologie Midi-Pyrénées pour le financement de l'opération et, notamment, M. Vaginay et M.-C. Jouffre pour le suivi du dossier, ainsi que F. Echassériaud de l'association Archéologies. Un grand merci à $\mathrm{S}$. Ducasse pour ses dessins. Nous remercions enfin G. Bariviera, C. Beauval, F. Chenu, F. LacrampeCuyaubère et F. Maksud pour leur indispensable aide logistique.

\section{REFÉRENCES BIBLIOGRAPHIQUES}

BALIJISTA S. (2006) - Du Magdalénien à l'Azilien en Quercy. Étude de l'industrie lithique des couches Vet IV de l'abri Murat (Rocamadour Lot). Fouilles $M$. Lorblanchet, mémoire de Master II, université de Toulouse-Le Mirail, ex. multigraph, 75 p.

BERGOUGNOUX F. (1887) - Les temps préhistoriques en Quercy, Cahors, 49 p.

CHALARD P. (1992) - L'abri de Peyrazet. Commune de Creysse (Lot), rapport de sondage, SRA Midi-Pyrénées, 15 p., ex. multigrapl.

COSTAMAGNO S., COCHARD D., FERRIE y.-G., LAROULANDIE V., BARBAZA M., CAZALS N., LANGLAIS M., VALDEYRON N., DACHARY M., GALOP D., MARTIN H., PHILIBERT' S. (2008) Nouveaux milieux, nouveaux gibiers, nouveaux chasseurs? Evolution des pratiques cynégétiques dans les Pyrénées du Tardiglaciaire au début du Postglaciàre, Bulletin de la Société préhistorique franģaise, $105(1)$, p. 17-27.

LANGLAIS M. (2007) - Dynamiques culturelles des sociétés magdaléniennes dans leurs cadres enviromementaux. Enquête sur 7000 ans d'évolution de leu's industries lithiques entre Rhône et Ebre, thèse de doctorat sous la cotutelle des universités de Toulouse-Le Mirail et Barcelone, ex. multigraph., 558 p.

LANGLAIS M., LAROULANDIE V. dir., avec la coll. de CHALARD P., COSTAMAGNO S., LE GALL O. (2007) - La grotte-abri de Peyrazet (Creysse, Lot). Le site, les restes archéologiques provenant du sondage d'évaluation et les problématiques de recherches, rapport annexe au dossier de demande d'autorisation d'opération, diffusion restreinte, SRA Midi-Pyrénées, ex. multigraph., 33 p.

LANGLAIS M., LAROULANDIE V. dir, avec la coll. de BRUXELLES L., CHALARD P., COCHARD D., COSTAMAGNO S DELFOUR G., KUNTZ D. LE GALL O, PÉTILLON J -M. QUEFFELEC A. (2008) - La grotte-abri de Peyrazet (Creysise, Lot, France). Occupations magdaléniennes et aziliennes entre causse et vallée du Haut-Quercy, rapport de foullle programmée annuelle, SRA Midi-Pyrénées, ex. multigraph.
LE GALl O. (1999) - Ichtyophagie et pêches préhistoriques. Ouelques données de l'Europe occidentale, thèse de Doctorat, université de Bordeatix I, ex. multigraph, p, 19]-193.

LE GALL O., DEMARS P.-Y., JARRY M., MAR'TIN H. (2007)-Saisons et proies : réflexions sur les notions de prédation(s) et de territoire(s), in M. Jatry dir., Cultures ef emironnements paléolithiques : mobilité et gestion des territoires des chasseurs-cueillews en Quercy, rapport de synthèse d'ACR, p. 307-325.

LEMOZIA. (1924) - Fouilies dans l'abri sous-roche de Murat, commune de Rocamadour, Lot. Bulletin de la Société préhistorique française, t. XXI, p. 17-58.

LORBLANCHET M. (1972) - Aperç sur le Magdalénien moyen et supérieur du Haut-Quercy, $C P F, X I X^{\circ}$ session, Auvergne, 1969, Soc. préhist. fr., p. 256-283.

LORBLANCHET M. (1989) - Caractères originaux dı Magdalénien du Quercy, in J.-P. Rigaud dir., Le Magdalénien en Europe, Colloque de Mayence, 1987, ERAUL, i. 38, p. 239-252.

LORBLANCHET M. (1996) - Du Magdalénien à l'Azilien en Quercy, La vie préhistorique, éd. Faton, p. 282-285.

PÉTILLON J.-M. (2006) -- Des Magdaléniens en arnes. Technologie des armatures de projectiles en bois de cervidé du Magdalénien supét rieur de la grotte d' lsturitz (Pyrénées-Atlantiques), Artefacts, 10, éd. Cedarc, Treignes, $302 \mathrm{p}$.

SÉrRONIE-VIVIEN M.-R, dir. (1995) - La grotte de Pégourié, Caniacdu-Causse (Lot). Périgordien-Badegoulien-Azilien-Áge du Bronze, Préhistoire quercynoise, sappl. 2, 334 p.

VIRÉ A. (1909) - Abri sous roche de la Rivière de Tulle près de Lacave, canton de Souillac, L'Anthropologie, t. XX, p. 273-282.

VIRÉ A. (1912) - Fouilles de la grotte du Pis de la Vache à La Forge, cre de Souillac, Lot, Bulletin de la Société préhistorique française, séance du 24 octobre 1912.

VIRÉ A., TEULIERE C (1927) - La Grolte-Bâtie (Crozo-Bastido) à Saint-Sozy (Lot), L'Anthropologie, t. 37, p. 449-458.

M. LANGLAIS' et V. LAROULANDIE ${ }^{2}$ avec la collaboration de L. BRUXELLES ${ }^{3}$, P. CHALARD ${ }^{3}$, D. COCHARD ${ }^{2}$, S. COSTAMAGNO ${ }^{3}$, G. DELFOUR, D. KUNTZ ${ }^{3}$, O. Le GALL ${ }^{2}$, J.-M. PETTLLON et A. QUEFFELEC ${ }^{2}$

1 : UMR 5608-TRACES

Université de Toulouse-Le Mirail et SERP-université de Barcelone Maison de la Recherche 5, allées A. Machado, 31058 Toulouse Cedex 9 matlang1@wanadoo.fr

2 : UMR 5199-PACEA, université de Bordeaux, B 18 Avenue des facultés, 33405 Talence Cedex

3 : UMR 5608-TRACES

Université de Toulouse-Le Mirail 5 allées A. Machado, 31058 Toulouse Cedex 9 